\title{
A New Spatio-temporal Event Model based on Multi-tuple for Cyber-Physical Systems
}

\author{
Jianhua Wang ${ }^{1}$, Lianglun Cheng ${ }^{2}$ and Jun Liu ${ }^{3}$ \\ Faculty of Automation, Guangdong University of Technology P. R. China \\ 123chihua@163.com ${ }^{1}$,llcheng@gdut.edu.cn ${ }^{2}$,liujun7700@163.com³
}

\begin{abstract}
Aiming to solve the problem of no well reflecting the spatial-temporal characteristic of CPS in current CPS event model, a new spatial-temporal event model based on multi-tuple for CPS is proposed in this paper.The basic idea of the new method is that we use the five tuples (object, time, space, event and property) to express a CPS event. As a result, our supposed model not only can more comprehensively reflect the spatial-temporal characteristic of CPS event, but also can reflect the dynamic change feature of external environment.The simulation results show that our proposed model scheme has a good performance in terms of reducing event average localization error rate and lowering event average timing error rate compared with other general event model methods.
\end{abstract}

Keywords: Event model; Spatio-temporal property; Cyber-Physical Systems (CPS); Multituple

\section{Introduction}

With the rapid development and relative mature in computer technology, communication technology, sensor technology and automatic control technology, Cyber-Physical Systems (CPS) [1] as a new intelligent system came into our life.

CPS get the help of perception capabilities, communication capabilities and computing capabilities embedded in physical devices to achieve the distributed perception, reliable data transmission, the intelligent information processing function in the external environment and to achieve the real-time control to physical process through feedback mechanisms. It has achieved to merge 3C technology (computing, communication and control) [2] into whole one and has a real-time, secure, reliable, high-performance characteristics. CPS brings us with a lot of convenience, but at the same time, it also brings us with a very important challenge: How to achieve a concurrency, timely and dynamic management between information systems and physical systems. That is the spatial-temporal characteristics of events [3]. Because CPS is an event-based system and all operations about computing and communications and control operations in the event system are processed based on event unit [4] and event can usually be manifested through its spatial-temporal features, the study of spatial-temporal feature in event has a very important role in the research of events and system design. In CPS, only when the spatio-temporal characteristics of event take some changes, event in CPS will occur, as CPS needs to make decisions based on events [5].

At present, some works have mainly been carried out to study event model concept and model design methods. In the definition of event concept aspect, Lu et al., [6] proposed a event definition method based on series of state changes; in the paper of [7], Auguston et al. proposed a event definition method based on execution behavior; Becker et al., proposed to 
define the individual behavior transformation as event in the article [8]. However, since the work mentioned above defined event only from an aspect of event, not with a comprehensive, holistic and dynamic view. These definitions above on event have some limitation and are not suitable to the event concept in CPS, which has a complex and changing environment. In event-based model design method aspects, because the event in CPS has its own some inherent properties, such as wide range of event definition, big heterogeneity of event composition, space distribution of event generation, sustainability and transience of event status, cause a lot of current event-based model design methods, for example, event model method based on data center [9] and event design methods based on Markov [10-12], are not directly suit to the event in CPS. The important reason for it is that the current design method of event model can not reflect environmental dynamic changes. So, how to design event model method which can reflect external dynamic changes of CPS becomes an important research focus of our current concern. In the current, some event models have been presented to describe the event property in CPS. Because the existing CPS event model does not or a little consider the dynamic change characteristics in CPS environmental, resulting in the current CPS event model can not fully reflect the spatial-temporal dynamic changes characteristics.

Arming to solving these problems above, a spatial-temporal event model based on multituple for CPS is proposed in this work. The basic idea of the new method is that we make use of five tuples (object, time, space, event and property) to express a CPS event. As a result, the new model not only can more comprehensively reflect the spatial-temporal characteristic of CPS event, but also can reflect the dynamic change feature of external environment. The simulation results show that our proposed model scheme has good performance in terms of reducing event average localization error rate and lowering event average timing error rate compared with some general event model method.

The rest of the paper is organized as follows: In Section 2, study state of CPS event model is reviewed. Event structure and principle based on multi-tuple are described in Section 3. The developed model is evaluated in Section 4. We conclude the paper in Section 5.

\section{Study state of CPS event model}

At present, some research works have been carried out to study CPS event models due to the increasingly growing importance of CPS. The current study state of CPS event model can be summarized as follows.

(1) Suppose a CPS event model based on the environmental objects, environmental objectives and the observed state of environment. In the paper [11], Cao et al., defined the environmental objects, environmental objectives and observed state of environment as a CPS event and established an auxiliary physical fusion system (ACPS) framework based on eventdriven. But the article neither took into account into change nature of outside world nor mention specific analysis on the event model.

(2) Suppose a layered temporal and spatial event model. The article [4] defined a new CPS architecture and a layered CPS event model based on the temporal and spatial properties of events. They proposed to uniformly express temporal-spatial CPS event using event property, event occurrence time and occurrence space. In this paper, they also proposed to use logical operators to combine different types of event conditions. Although they analyzed their proposed model in this paper, it did not verify their supposed event model.

(3) Suppose an event model based on concept lattice. In the work of [13], a CPS event model based on event type, internal properties and external properties is uniformly 
represented. Tan et al., thought that a CPS event model can be divided into three components: event type, internal properties and external properties. They also believed that the event type, spatio-temporal properties of the event and the observer can be characterized by the internal and external properties together. In this work, they also analyzed and verified their proposed event model. The defect of this paper rested that the supposed event model did not reflect the changing degree and changing result of external environment, such as quantitative change or qualitative change.

(4) Suppose an Adaptive Discrete Event (ADE) model. Yue et al., [14] presented a ADE model and made use of Discrete Event Calculus (DEC) to solve the problem of possible inherent inconsistencies in composition rules which are specified by first order logic. But this model was not suitable for continuous events and it also did not think over the dynamically changing state of external environment.

(5) Suppose an event model based on the physical state factors. In the work of [15], Chen et al., put forward an event model based on physical state. The article defined event model to depict the changes in the physical world by a physical state variable. It has a certain rationality, but not comprehension. Since the event model in the paper do not reveal the nature of external change from constituent tuples of CPS event.

To address the problem above, in this paper, we propose a new spatial-temporal event model based on five tuples (object, time, space, event and property) for CPS on the basis of the structure analysis of current CPS event model. Our supposed new model not only can more comprehensively reflect the spatial-temporal characteristic of CPS event, but also can reflect the dynamic change feature of external environment, such as changing degree and changing result.The simulation results improve the feasibility of our method.

\section{Event Structure and Principle based on Multi-tuple}

In this section, we formally illustrate the event structure and principle based on multi-tuple in CPS. It mainly include four part: CPS event instance based on multi-tuple, CPS observer event instance based on multi-tuple, global function of CPS event instance based on multituple and case study of CPS event instance based on multi-tuple.

\subsection{CPS event instance based on multi-tuple}

The CPS event model requires not only reflect time and spatial characteristics of the heterogeneous distribution node, but also need to reflect the dynamic changes of physical environment in the CPS system. In this paper, we propose a spatial-temporal event model based on multi-tuple for CPS to solve the problem of no well reflection dynamic changes nature of external environment for most current CPS event model. Our supposed model not only can reflect the dynamic nature of external environment, but also can describe the change degree and change results, more comprehensively reflecting the spatial-temporal characteristic of CPS event.A CPS event instance in this work refers to the state transition and state changes of spatial-temporal event. The definition of CPS event model based on multituple is shown in (1) as follows.

\section{Definition 1(CPS Event Instance)}

$$
m_{c p s}=\mathrm{E}\left(B^{g}, \mathrm{~T}^{g}, \mathrm{~L}^{g}, U^{g}\right) @(B, \mathrm{~T}, \mathrm{~L}, O)
$$


Where

E : represent the event type of the CPS event instance;

$B^{g}$ : represent the object at which the CPS event instanc act;

$\mathrm{T}^{g}$ : represent the time at which the CPS event instanc is generated.

$\mathrm{L}^{g}$ : represent the location at which the CPS event instanc is generated.

$U^{g}$ : represents a finite set of properties of the CPS event instanc.

$B$ : represent the object at which the CPS event instanc act;

$\mathrm{T}$ : represent the time at which the CPS event instanc is observed to occur.

$\mathrm{L}$ : represent the location at which the CPS event instanc is observed to occur.

$O:$ is the observer of the CPS event instance.

The interrelationships in composed tuples for new CPS event model are shown as in Figure 1.

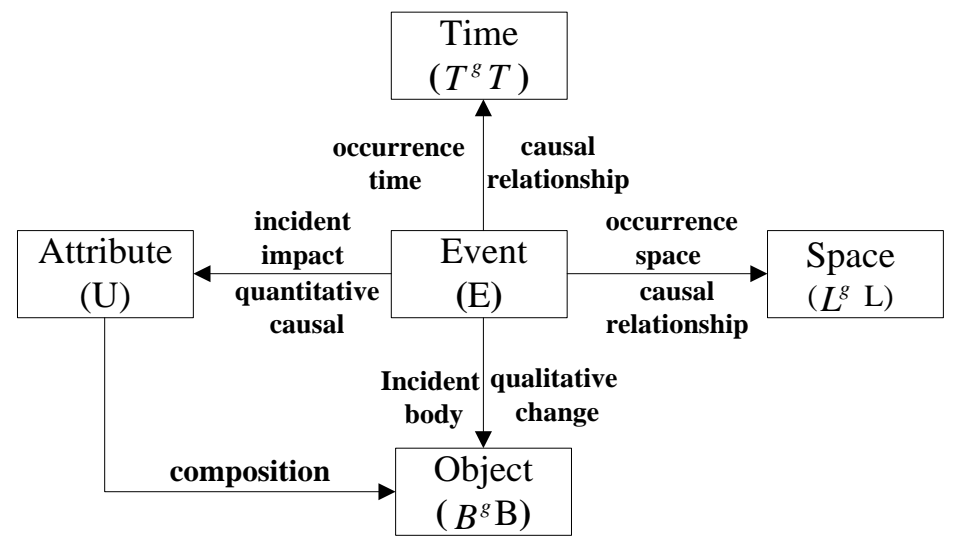

Figure 1. The interrelationships in composed tuples for new CPS event model

From Figure 1, we can see that our supposed CPS event can be fully expressed by five composed tuples and there are some important interrelationships in the five composed tuples above, such as causal relationship, quantitative relationship and qualitative relationship. In this paper, the dynamic change features of external environment in CPS are mainly reflected by property and object tuple. The property and object tuple in our CPS event model can describe changing degree and changing result occurred at a period of time or space. Since property tuple can reflect the small change and represent quantitative change, while object could reflect big change and represent the qualitative change.

In formula (1), thanks to explicit timing constraints, the temporal property $\mathrm{T}^{g}$ and $\mathrm{T}$ stand for real-clock timestamps instead of logical-clock timestamps and they are given in the form of a time interval, such as $[a, b]$ or $(a, b],(a, b),[a, b)$. When $a=b$, the event is an instant event. Similarly, the spatial tuple $\mathrm{L}^{g}$ and $\mathrm{L}$ in (1) are given in the form of $((\mathrm{x}, \mathrm{y}, \mathrm{z}), \mathrm{r})$, where $(\mathrm{x}, \mathrm{y}, \mathrm{z})$ is the relative geographical coordinates with respect to the observer $\mathrm{O}, \mathrm{r}$ indicates the radius of the event. When $r=0$ means a point event, $r>0$ means a field event.

With the interpretation and limitation above, formula (1) can be written in the form of formula (2) as follows,

$$
m_{c p s}=E \underbrace{\left(B^{g},\left[t_{1}^{g}, t_{2}^{g}\right],\left[\left(x^{g}, y^{g}, z^{g}\right), r^{g}\right], U^{g}\right)}_{\text {event information produced by CPS observers }} @ \underbrace{\left(B,\left[t_{1}, t_{2}\right],[(x, y, z), r], O\right)}_{\text {event information of CPS observers }}
$$


As is seen by the formula (2) above, the definition of our CPS event model consists of two parts: the event information produced by CPS observers and event information of CPS observes. The first section of formula (2) is the event information produced by CPS observers, which can be gotten by the observing CPS observer; while the second portion of formula (2) is the event information of CPS observers, which can be obtained by observing information of environment. There is a highly application-dependent feature in event information produced by CPS observers section for our CPS event and it represents uncertain properties that all CPS events have, while there is application-independent in the event information of CPS observes and it represents fixed properties that all CPS events have. A major difference between our model and traditional event models lies in the observed event information part.

In short, formula (1) describes an event instance $m_{c p s}$ of event type $\mathrm{E}$ with object $B$ and property $U^{g}$ observed by $O$. The event instance $m_{c p s}$ is observed to occur at time $\mathrm{T}$ and location $\mathrm{L}$ with respective to the observer location. Then, the event instance is generated at time $\mathrm{T}^{g}$ and location $\mathrm{L}^{g}$ with respect to the observer.

\subsection{Observer of CPS event instance based on multi-tuple}

In cyber-physical systems, the observer $\mathrm{O}$ is also as an event instance, it can dynamically join or leave a CPS and can are reported as events by any other observer. The observer event instance in our event model is defined as follows:

\section{Definition 2 (CPS observer event instance)}

$$
m_{c p s}=\mathrm{E}\left(B^{g}, \mathrm{~T}^{g}, \mathrm{~L}^{g}, U^{g}\right) @(B, \mathrm{~T}, \mathrm{~L}, O)
$$

Where

$B^{g}$ : represent the object at which the specific observer act.

$U^{g}$ : represents a finite set of property of the observer event related to the global observer. It includes the set of event generation rules related to the observer, the set of event types that this observer can generate, and so on.

$\mathrm{T}^{g}$ : represent the time at which the observer event is generated.

$\mathrm{L}^{g}$ : represent the location at which the observer event is generated.

$B$ : represent the object at which the observer event act.

$\mathrm{T}$ : represent the time at which the observer event is observed to occur.

$\mathrm{L}$ : represent the location at which the observer event is observed to occur.

$O_{\perp}$ : is the global observer and it represent $O_{c p s}$ in CPS.

In formula (3), the role of global observer $O_{\perp}$ is to analyze system, so it is necessary to provide a common frame of reference in analysis system. There is only one global observer $O_{\perp}$ in each specific CPS system. We set that the location of observer $O_{\perp}$ is the system origin and the time interval of observer $O_{\perp}$ is defined as the system's life span. The definition of global observer $O_{\perp}$ is shown in formula (4)

$$
O_{\perp}=E_{s}\left(\left(B^{g},[0, \infty],[(0,0,0), 0], U^{g}\right) @\left(B,[0, \infty],[(0,0,0), \infty], O_{c p s}\right)\right.
$$

where $O_{c p s}$ denotes the CPS system itself. 
In a CPS system, though mobile observers need to determine the granularity of spatial accuracy required, the process of mobile observers is similarly as CPS event observers above.

\subsection{Global function of CPS event instance based on multi-tuple}

In a CPS system, there are usually a variety of different types of objects and different types of objects produce the different types of event. The characteristics of inherent heterogeneity and spatial distribution of CPS system result in the non-uniformity of observer and some difficulty in comparing different CPS event. So in order to easily compare two different CPS events in time, location, property or combine easily CPS events, we need to provide a global function and make use of it to convert the located events observed by a different observers into a global events observed by a unified observers. In this paper, the definitions for global function are as follows:

\section{Definition 3 (CPS global function)}

Given an $m_{c p s}$ event observed:

$$
m_{c p s}=E\left(B^{g},\left[t_{1}^{g}, t_{2}^{g}\right],\left[\left(x^{g}, y^{g}, z^{g}\right), r^{g}\right], U^{g}\right) @\left(B,\left(t_{1}, t_{2}\right),[(x, y, z), r], m_{s}\right)
$$

And an observers event

$$
m_{s}=E_{s}\left(B_{s}^{g},\left[t_{s}^{g}, \infty\right),\left[\left(x_{s}^{g}, y_{s}^{g}, z_{s}^{g}\right), 0\right], U_{s}^{g}\right) @\left(B_{s},\left[t_{s}, \infty\right),\left[\left(x_{s}, y_{s}, z_{s}\right), 0\right], O_{c p s}\right)
$$

the global function $\mathrm{G}: \mathrm{M} \rightarrow \mathrm{M}$ is defined by:

$$
\begin{aligned}
& m_{g l o}=G\left(m_{c s p}\right) \\
& =E\left(B^{g} B_{s}^{g},\left[t_{s}+t_{1}^{g}-t_{s}^{g}, t_{s}+t_{2}^{g}-t_{s}^{g}\right],\left[\left(x_{s}+x^{g}-x_{s}^{g}, y_{s}+y^{g}-y_{s}^{g}, z_{s}+z^{g}-z_{s}^{g}\right), r^{g}\right], U^{g}\right) \\
& @\left(B B_{s},\left[t_{s}+t_{1}-t_{s}, t_{s}+t_{2}-t_{s}\right],\left[\left(x_{s}+x-x_{s}^{g}, y_{s}+y-y_{s}^{g}, z_{s}+z-z_{s}^{g}\right), r\right], O_{c p s}\right) \\
& =E\left(B_{g l o}^{g},\left[t_{s}+t_{1}^{g}-t_{s}^{g}, t_{s}+t_{2}^{g}-t_{s}^{g}\right],\left[\left(x_{s}+x^{g}-x_{s}^{g}, y_{s}+y^{g}-y_{s}^{g}, z_{s}+z^{g}-z_{s}^{g}\right), r^{g}\right], U^{g}\right) \\
& @\left(B_{g l o},\left[t_{s}+t_{1}-t_{s}, t_{s}+t_{2}-t_{s}\right],\left[\left(x_{s}+x-x_{s}^{g}, y_{s}+y-y_{s}^{g}, z_{s}+z-z_{s}^{g}\right), r\right], O_{c p s}\right)
\end{aligned}
$$

\subsection{Case study of CPS event instance based on multi-tuple}

In order to better illustrate the event structure and its application based on multi-tuple, we now take the temperature and light detection of an indoor room in complex industry for an example to illustrate our practical application for the formula (1-3) above. For explaining the problem simply, we only assume that there are two types of sensors: temperature sensor and light sensor installed in an indoor room to monitor room environmental condition. Temperature sensor is used to detected temperature intensity of indoor room, while light sensor is used to detected light intensity of indoor room. We assume that the temperature sensor can generate temperature event and the light sensor could produce a light event.

In this example above, due to the temperature sensor and the light sensor itself act as an observer, they themselves are also CPS event instances and can generate the temperature sensor event and light sensor event against CPS system respectively. Other some main parameters are set for the scenario in Table 1. 
Table 1. Other various conditions set in Scene

\begin{tabular}{|c|c|c|}
\hline paremater & $\begin{array}{c}\text { temperature } \\
\text { sensor }\end{array}$ & light sensor \\
\hline install at global point location & $((5,5,5), 0)$ & $((6,6,6), 0)$ \\
\hline start in global point time & {$[5, \infty]$} & {$[6, \infty]$} \\
\hline initialized relative location & $((0,0,0), 0)$ & $((0,0,1), 0)$ \\
\hline initialized relative time & {$[0, \infty]$} & {$[0, \infty]$} \\
\hline observed event occurrence location & 5 disdance & 6 disdance \\
\hline event instance generation location & 12 disdance & 13 disdance \\
\hline observed event occurrence time & 8 disdance time & 9 disdance \\
\hline event instance generation time & 12 disdance time & 13 disdance time \\
\hline
\end{tabular}

Combining the example function and formula (1) (3) (5) above, we can get the mathematical expression of temperature sensor event instance, light sensor event instance, temperature event instance and light event instance as follows.Formula (6) and (7) are the temperature sensor event instance and light sensor event instance respectively.

$$
\begin{aligned}
& m_{\text {temperature sensor }} \\
& \left.=E_{\text {temperature sensor obseve }}\left(B_{\text {temperature sensor }},[0, \infty),((0,0,0), 0)\right),\left(h_{t}, p_{\text {temperature }}\right)\right) @ \\
& \left(B_{\text {temperature sensor }},[5, \infty),((5,5,5), 0), \mathrm{O}_{\mathrm{cps}}\right) \\
& m_{\text {light sensor }} \\
& =E_{\text {light sensor obseve }}\left(B_{\text {light sensor }},[0, \infty),((0,0,1), 0),\left(h_{l}, p_{\text {light }}\right)\right) @ \\
& \left(B_{\text {light sensor }},[6, \infty),((6,6,6), 0), \mathrm{O}_{\mathrm{cps}}\right)
\end{aligned}
$$

In formula (6), $\mathrm{m}_{\mathrm{te}}$ is an temperature observer event instance; $E$ indicates that it is a temperature observer event type; $B_{\text {temperature sensor }}$ is the object at which the $\mathrm{O}_{\text {cps }}$ act is temperature; $[0, \infty)$ represent initial timer of the temperature sensor $\mathrm{m}_{\text {temperature sensor }} ;((0,0,0), 0)$ represent initial location for the temperature sensor $\mathrm{m}_{\text {temperature sensor }}$; the $h_{t}$ and $p_{\text {tenper at ure }}$ in property tuple represent set of event generation rules related to $m_{\text {temperature sensor }}$ and set of temperature event that $m_{\text {temperature sensor }}$ can generate respectively; $[5, \infty)$ represent the time at which the $m_{\text {temperature sensor }}$ is observed to occur; $(5,5,5), 0)$ represent the location at which the $m_{\text {temperature sensor }}$ is observed to occur; $\mathrm{O}_{\text {cps }}$ represent the global observer.

In formula (7), $m_{\text {light sensor }}$ is an light observer event instance; $E_{\text {light sensor obseve }}$ indicates that it is a light observer event type; $B_{\text {light sensor }}$ represent the at object which the $\mathrm{O}_{\mathrm{cps}}$ act is light sensor; $[0, \infty)$ represent initial timer of the light sensor $m_{\text {light sensor }} ;((0,0,1), 0)$ represent initial location for the light sensor $m_{\text {ligh sensor }}$; the $h_{l}$ and $p_{\text {light }}$ in property tuple represent set of event generation rules relate to $m_{\text {light sensor }}$ and set of light event that $m_{\text {light sensor }}$ can generate respectively; [6, $\infty$ ) represent the time at which the $m_{\text {light sensor }}$ is observed to occur; $\left.(6,6,6), 0\right)$ represent the location at which the $m_{\text {light sensor }}$ is observed to occur; $\mathrm{O}_{\text {cps }}$ represent the global observer. 
The temperature event instance that is generated by the temperature sensor and the light event instance that is generated by the light sensor can be represented in formula (8) and (9) as follows:

$$
\begin{aligned}
& m_{\text {tenper at ure }}=E_{\text {tenper at ure det ecti on }}\left(B_{\text {tenper at ure }},[25,25],((0,0,0), 0), U_{\text {tenper at ure int ensi ty }}\right) \\
& @\left(B_{\text {tenper at ure }},[13,13],((0,0,0), 5), m_{\text {tenper at ure sensor }}\right) \\
& m_{1 \text { i ght }}=E_{\text {li ght det ect i on }}\left(B_{1 \text { i ght }},[26,26],((0,0,1), 0), U_{\text {tenper at ure int ensi ty }}\right) \\
& @\left(B_{\text {li ght }},[14,14],((0,0,0), 6), m_{1 \text { i ght sensor }}\right)
\end{aligned}
$$

In formula (8), $m_{\text {tenperat ure }}$ is the temperature event instance; $E_{\text {tenper at ure det ecti on }}$ represent temperature event type; $B_{\text {temperature }}$ represent the object at which the observer $m_{\text {tenper at ure sensor }}$ act; $[25,25]$ and $((0,0,0), 0)$ represent $m_{\text {tenperature }}$ instance is generated at time $25 \mathrm{~s}$ and location $((0,0,0), 0)$ relative to the $m_{\text {tenper at ure sensor }}$, since it is generated by the $m_{\text {tenper at ure sensor }} ; U_{\text {tenper at ure intensity }}$ represent temperature intensity property observed; In formula (8), the temperature sensor also reports that the $m_{\text {tenperature }}$ is observed to occur at sensor time $[13,13]$ and within $((0,0,0), 5)$ units of its location. The $m_{\text {tenperat ure sensor }}$ indicates that the event instance $m_{\text {tenper at ure }}$ is generated by $m_{\text {tenper at ure sensor }}$.

In formula (9), $m_{\text {light }}$ is the light event instance; $E_{\text {light detection }}$ represent light event type; $B_{\text {light sensor }}$ represent the object at which the observer $m_{\text {light sensor }}$ act; $[26,26]$ and $((0,0,1), 0)$ represent $m_{\text {light }}$ instance is generated at time $26 \mathrm{~s}$ and location( $\left.(0,0,1), 0\right)$ relative to the $m_{\text {light sensor }}$ since it is generated by the $m_{\text {light sensor }} ; U_{\text {light intensity }}$ represent light intensity property observed. In formula (9), The light sensor also reports that the $m_{\text {light }}$ is observed to occur at sensor time $[14,14]$ and within $((0,0,0), 6)$ units of its location. The $m_{1 \text { ight sensor }}$ indicate that the $m_{1 \mathrm{ight}}$ is generated by $m_{1 \mathrm{~g} \text { ght sensor }}$.

Formula (10) and (11) are the global temperature sensor event instance and light sensor event instance respectively.

$$
\begin{aligned}
& m_{\text {tenper at ure }}=E_{\text {tenperat ure det ecti on }}\left(B_{\text {tenper at ure }},[30,30],((5,5,5), 0), U_{\text {tenper at ure int ensi ty }}\right) \\
& @\left(B_{\text {tenper at ure }},[18,18],((5,5,5), 5), O_{c p s}\right) \\
& m_{1 \text { i ght }}=E_{\text {li ght det ecti on }}\left(B_{1 \text { i ght }},[32,32],((6,6,0), 0), U_{\text {li ght int ensi ty }}\right) \\
& @\left(B_{\text {li ght }},[20,20],((6,6,5), 6), O_{c p s}\right)
\end{aligned}
$$

From formula (10) and (11), we can see cleanly that, with the help of the globalization function, the $m_{\text {tenper at ure }}$ and $m_{1 \mathrm{ight}}$ changes its observer from local observer $m_{\text {temperature sensor }}$ to the global observer $O_{c p s}$ and from local observer $m_{\text {light sensor }}$ to the global observer $O_{c p s}$ respectively. Correspondingly, the event occurrence time, location and the event generation time, location of $m_{\text {tenperature }}$ and $m_{1 \text { ight }}$ should be also converted into the global observer's perspective. 


\section{Experimental results and analysis}

In this paper, an event model based on multi-tuple for CPS has been presented. In order to evaluate the efficiency of the proposed model, we performed some experiments on Microsoft Windows 7 operating systems, AMD A6-3420M 4 core CPU Processor, 2G memory, 500G Hard disk, Tossim tool. Where Tossim tool, which is based on CPS event simulator (CPSim), is made up of two parts: physical event simulation (PES) and cyber event simulations (CES). The main function of PES is to simulate the physical phenomenon of interest and it is realized by Python, while the main function of CES is to simulate the behaviors of sensors and observers in the CPS and its realization is in Python.

In this paper, our experiment scene is that a small ball moves back and forth for 60 s on a path length of $10 \mathrm{~m}$ with a $4 \mathrm{~m}$ long test path in the middle of it at different rates. During the experiment, if the time that samll ball goes through the test path of $4 \mathrm{~m}$ above is more than 2 $\mathrm{s}$, small ball will trigger a ball event. Experiment detects the performance of ball event localization error rate and ball event timing error rate compared with currently three general event model method (event model based on time, event model method based on space and event model based on time-space). In our experiments, we use the Cricket sensor to measure the distance of the small ball move and to judge whether the movement of small ball is in the test area. We set the sampling speed at $0.2 \mathrm{~s}$ for Cricket. Time is counted by using precision timer. In order to reduce the experimental error due to the randomness, the experimental data is obtained on the basis of 10 times repeated test. The ball event localization error rate and ball event timing error rate in the paper is defined as follows:

average localization error rate of ball event

$=\underline{\text { ball event number which donot satisfy detection localization requirement }}$

total number of ball event

average timing error rate of ball event

$=\underline{\text { bal I event number whi ch donot sat i sf } \mathrm{y} \text { det ect } \mathrm{i} \text { on } \mathrm{t} \mathrm{i} \text { ming requi rement }}$

t ot al nunber of ball event

\subsection{Test average localization error rate of ball event}

Figure 2 is the experimental comparison result in the average localization error rate of ball event for our proposed method and other two event model methods.

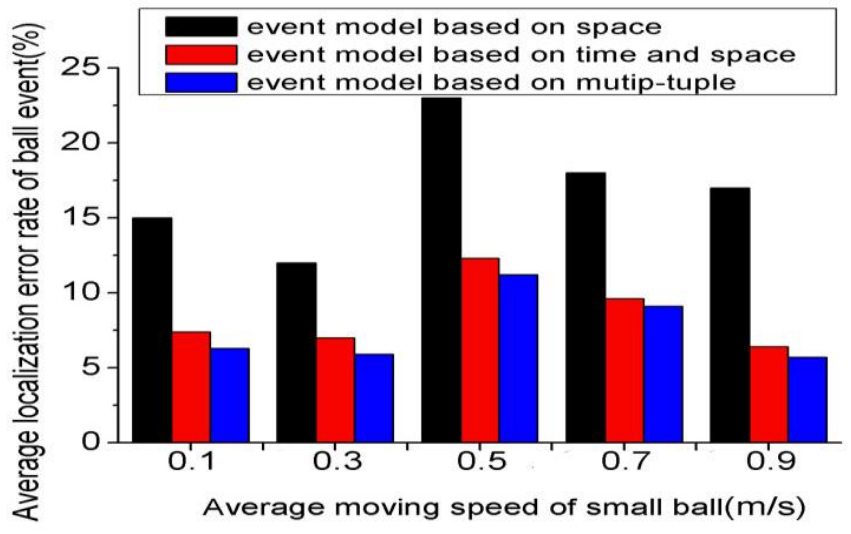

Figure 2. Comparison result in average localization error rate of ball event 
From Figure 2, we can see that, our supposed event model has good performance in average localization error rate of ball event compared with the other two event models. It is followed by the event model based on time and space. Event models based on space shows the worst localization performance in three event models.

The main reasons for the phenomenon above are that:

On the one hand, the event model based on space can reflect ball event only from the occurrence space of ball event. The event model based on time and space can reflects ball event from the two occurrence space and occurrence time of ball event, which can improve in localization accuracy of ball event compared with event model based on space.

On the other hand, since our event model add object and property tuple to express the localization change feature of ball event, which can help to improve localization accuracy of ball event by interrelationships existed in composed tuples in our event model compared with event model based on time and space. Except that it has a good performance in average localization error rate, our supposed method can also describe some change feature of ball event in $4 \mathrm{~m}$ test path, such as the changing degree and changing results of ball event, therefore, more comprehensively reflecting the spatial-temporal feature of ball event.

\subsection{Test average timing error rate of ball event}

Figure 3 is an experimental comparison result in average timing error rate of ball event for our proposed method and other two common event model methods.

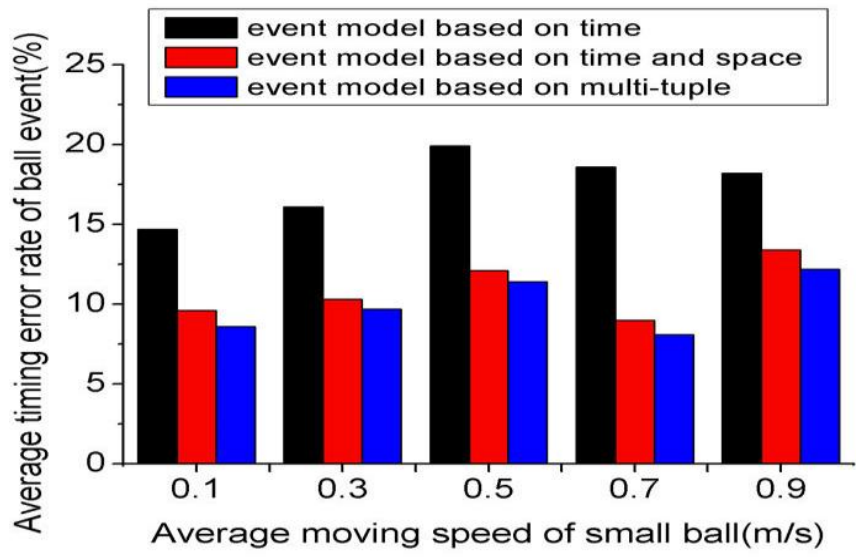

Figure 3. Comparison result in average timing error rate of ball event

From Figure 3, we can observe that our proposed event model shows lower average timing error rate in three methods above. Event model based on time and space follows it. Event models based on time has the biggest timing error rate.

The main reasons for them lie that:

One the one hand, the event model based on time describes ball event only from the occurrence time of ball event. The event model based on time and space can describe ball event from the two occurrence space and time of ball event, which can help to reduce the timing error rate of ball event compared with event model based on time.

One the other hand, our event model based on multi-tuple makes use of interrelationships existed in composed tuples in event model to describe ball event from object, time, space, event and property aspects, which can help to improve the timing accuracy of ball event 
compared with other methods. Except that it has a good performance in average timing error rate, our supposed method can also describe some time change feature of ball event in $2 \mathrm{~s}$, such as the changing degree and changing results of ball event, thus more comprehensively reflect the spatial-temporal feature of ball event compared with event model based on time and space in our event model.

\section{Conclusion}

In this paper, a spatial-temporal event model based on multi-tuple for CPS is proposed to solve the problem of no well reflecting the spatial-temporal characteristic of CPS in most current CPS event model. In our scheme, we uses the union of five tuples, object, time, space, events and property, to describe a CPS event model, as a result, our model not only can more comprehensively reflect the spatial-temporal characteristic of CPS, but also can reflect the dynamic change feature of external environment, such as describing the changing degree and changing results. The simulation results show that our proposed model scheme has good performance in terms of reducing event average localization error rate and event average timing error rate compared with some general event model methods.

\section{Acknowledgements}

The authors would like to thank the anonymous reviewers for their constructive opinions in improving this paper. The work was supported by the Joint Funds of the National Natural Science Foundation of China and Guangdong Natural Science Foundation ( No.U2012A002D01) ; Key Projects of National Natural Science Foundation of China (No.U2012A002D01); The Scientific Research Project of Guangzhou City (No: 12C42111582) and (No: 2012Y-00041).

\section{References}

[1] R. Pathetic and H. Gill, "Cyber-physical systems", The Impact of Control Technology. Washington D. C. IEEE, vol. 42, no. 3, (2011), pp. 161-166

[2] Vincentelli, K. Shin, J. Stankovic, J. Sztipanovits, W. Wolf, W. Zhao, "Cyber-Physical Systems, Executive Summary”, CPS Steering Group,Washington $\quad$ D.C., $\quad$ (2008) March 6, http://www.nsf.gov/pubs/2008/nsf08611/nsf08611.htm.

[3] C. Talcott, "Cyber-Physical Systems and Events" In Software Intensive Systems and New Computing Paradigms, vol. 5380, (2008), pp. 101-115.

[4] Y. Tan, M. C. Vuran and S. Goddard, "Spatio-Temporal Event Model for Cyber-Physical Systems", The 29th IEEE International Conference on Distributed Computing Systems Workshops, (2009) June 22-26, Quebec, Canada.

[5] S. Lu, J. Huang and B. Q. Yin, "Modeling and simulation of the admission control of VOD based on POMDP”, Journal of University of Science and Technology of China, vol. 39, no. 9, (2009), pp. 984-989.

[6] X. -R. Cao and J. Zhang, "Event-Based Optimization of Markov Systems", IEEE Transaction on automatic control, vol. 53, no. 4, (2008).

[7] M. Auguston, "Software architecture built from behavior models", ACM SIGSOFT Software Engineering Note, vol. 34, no. 5, (2009).

[8] R. Becker, S. Zilberstein and V. Lesser, "Decentralized Markov Decision Processes with Event-Driven Interactions", AAMAS '04 Proceedings of the Third International Joint Conference on Autonomous Agents and Multiagent Systems, (2004) July 19-23, New York, USA.

[9] X. Li, Y. Qiao, X. Li and H. A. Wang, "Real-Time ECA Rule Reasoning in Active Database", Journal of Computer Research and Development, vol. 47, Suppl., (2010).

[10] X. -R. Cao and J. Zhang, "Event-Based Optimization of Markov Systems", IEEE Transactions on automatic control, vol. 53, no. 4, (2008).

[11] X. -R. Cao, "Basic Ideas for Event-Based Optimization of Markov Systems", Discrete Event Dynamic Systems: Theory and Applications, vol. 15, (2005), pp. 169-197. 
[12] F. Makedon, Z. Le, H. Huang and E. Becker, "An event driven framework for assistive CPS environments", ACM SIGBED Review-Special Issue on the 2nd Joint Workshop on High Confidence Medical Devices, Software and Systems (HCMDSS) and Medical Device Plug-and-Play (MD PnP) Interoperability, vol. 6, no. 2, (2009).

[13] Y. Tan, M. C. Vuran, S. Goddard, Y. Yu, M. Song and S. Ren, "A Concept Lattice-based Event Model for Cyber-Physical Systems", The 1st CM/IEEE International Conference on Cyber-Physical Systems (ICCPS), (2010) April 13-15, Stockholm, Sweden.

[14] Y. Ke, W. Li, R. Shangping, M. Xufei and L. Xiangyang, "An Adaptive Discrete Event Model for CyberPhysical System", The First Analytic Virtual Integration of Cyber-Physical Systems Workshop, (2010) November 30, San Diego, California, USA.

[15] C. Li na, H. Hongbin and S. Deng, "Research on CPS Spatio-Temporal Event Model based on the State", Computer Science \& Education. The 6th International Conference on Computer Science \& Education, (2011), August 3-5, SuperStar Virgo, Singapore.

\section{Authors}

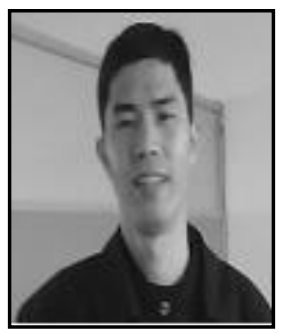

\section{JianHua Wang}

He was born on February 6, 1982 in GuangDong, China. He received his B.S degree in Electronic Information Science and Technology from ShaoGuan University, GuangDong, China, in 2006. Currently he is pursuing Ph.D degree in Control Science and Engineering at Guangdong University of Technology. His research interests include $3 \mathrm{G}$ wireless video transmissions, cyber-physical systems and wireless sensor networks.

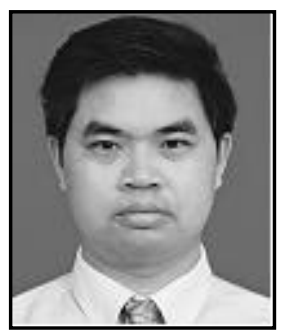

\section{LiangLun Cheng}

He was born on August 22, 1964 in HuBei. He received his M.S and Ph.D degrees from Huazhong University of Science and Technology, HuBei, China in 1992 and Chinese academy of Sciences JiLin, china in 1999 respectively. He is a Prof and doctoral supervisor of Guangdong University of Technology. His research interests include $3 \mathrm{G}$ wireless video transmissio, RFID and WSN, IoT and CPS, production equipment and automation of the production process, embedded system, the complex system modeling and its optimization control, software of automation and information, etc.

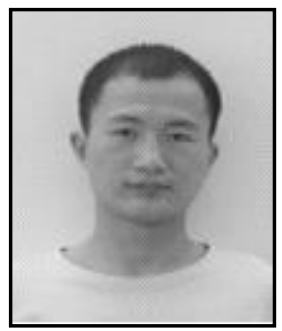

\section{Jun Liu}

He was born on October 11, 1986 in Hubei, China. He received his M.S degree in Control Science and Engineering from Guangdong University of Technology, Guangdong, China, in 2012. Currently he is pursuing Ph.D degree in Control Science and Engineering at Guangdong University of Technology. His research interests include $3 \mathrm{G}$ wireless video transmissions, cyber-physical systems and wireless sensor networks. 